\title{
Method of separation between light and heavy groups of primary CR nuclei by LDF of Cherenkov light in the range $300-3000 \mathrm{TeV}$
}

\begin{abstract}
A Sh Elshoukrofy ${ }^{1,15}$, L Sveshnikova ${ }^{1}$, E Postnikov¹, I Astapov9, P Bezyazeekov², V Boreyko ${ }^{10}$, A Borodin ${ }^{10}$, M Brueckner ${ }^{8}$, N Budnev ${ }^{2}$, A Chiavassa4 , A Dyachok ${ }^{2}$, 0 Fedorov ${ }^{2}$, A Gafarov ${ }^{2}$, A Garmash ${ }^{11}$, N Gorbunov ${ }^{10,16}$, V Grebenyuk ${ }^{10,16}$, 0 Gress $^{2}$, T Gress ${ }^{2}$, 0 Grishin², A Grinyuk ${ }^{10}$, D Horns ${ }^{6}$, A Ivanova², N Kalmykov ${ }^{1}$, Y Kazarina2, V Kindin?9, S Kiryuhin², P Kirilenko11, R Kokoulin9 , K Kompaniets9, E Korosteleva ${ }^{1}$, V Kozhin ${ }^{1}$, E Kravchenko ${ }^{11,12}$, M Kunnas $^{6}$, L Kuzmichev ${ }^{1}$, Yu Lemeshev ${ }^{2}$, V Lenok², B Lubsandorzhiev'1,3, N Lubsandorzhiev', R Mirgazov ${ }^{2}$, R Mirzoyan ${ }^{5,2}$, R Monkhoev ${ }^{2}$, R Nachtigall', E Osipova², A Pakhorukov², M

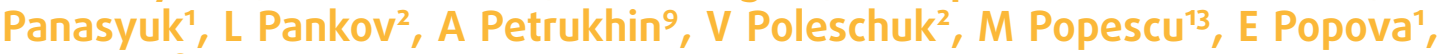
A Porelli, V Prosin'1, V Ptuskin7, E Rjabov², G Rubtsov³, A Pushnin², Y Sagan $^{10,16}$, B Sabirov ${ }^{10}$, V Samoliga ${ }^{2}$, Yu Semeney ${ }^{2}$, A Silaev ${ }^{1}$, A Silaev (junior) ${ }^{1}$, A Sidorenkov3 , A Skurikhin ${ }^{1}$, V Slunecka ${ }^{10}$, A Sokolov ${ }^{11,12}$, C Spiering ${ }^{8}$, V Tabolenko², B Tarashansky², A Tkachenko ${ }^{10}$, L Tkachev ${ }^{10,16}$, M Tluczykont ${ }^{6}$, R Wischnewski ${ }^{8}$, A Zagorodnikov², D Zhurov², V Zurbanov², I. Yashin9 (TAIGA collaboration), and A A Darwish ${ }^{14}$, and H A Motaweh ${ }^{15}$
\end{abstract}

A Sh Elshoukrofy

abeershehatamahmoud@

yahoo.com

L Sveshnikova

tfl10@mail.ru

E Postnikov

evgeny.post@gmail.com

Received: 25 December 2017 Accepted: 2 February 2018 Published: 9 April 2018

Publishing services provided by Knowledge E

(c) A Sh Elshoukrofy et al. This article is distributed under the terms of the

Attribution License, which permits unrestricted use and redistribution provided that the original author and source are credited.

Selection and Peer-review under the responsibility of the ICPPA Conference Committee.

\section{G OPEN ACCESS}

\author{
${ }^{1}$ Skobeltsyn Institute of Nuclear Physics MSU, Moscow, Russia \\ ${ }^{2}$ Institute of Applied Physics ISU, Irkutsk, Russia \\ ${ }^{3}$ Institute for Nuclear Research of RAN, Moscow, Russia \\ ${ }^{4}$ Dipartimento di Fisica Generale Universiteta di Torino and INFN, Torino, Italy \\ ${ }^{5}$ Max-Planck-Institute for Physics, Munich, Germany \\ ${ }^{6}$ Institut für Experimentalphysik, University of Hamburg, Germany \\ 7IZMIRAN, Moscow, Russia \\ ${ }^{8}$ DESY, Zeuthen, Germany \\ ${ }^{9}$ National Research Nuclear University MEPhl, Moscow, Russia \\ ${ }^{10}$ JINR, Dubna, Russia \\ ${ }^{11}$ Novosibirsk State University, NSU, Novosibirsk, Russia \\ ${ }^{12}$ Budker Institute of Nuclear Physics SB RAS, Novosibirsk, Russia \\ ${ }^{13}$ ISS, Bucharest, Romania \\ ${ }^{14}$ Faculty of science, Alexandria University,Alexandria, Egypt \\ ${ }^{15}$ Faculty of science, Damanhur University, Damanhur, Egypt \\ ${ }^{16}$ Dubna State University, Dubna, Russia
}

\section{Abstract}

The problem of chemical composition below the knee in the cosmic-ray energy spectrum has not yet been solved due to low statistics collected from direct experiments. In the HiSCORE experiment the lateral distribution functions (LDF) of Cherenkov light of EASs with energy greater than hundreds of TeV can be measured in detail for millions of individual events. A full steepness of LDF is sensitive to the depth of shower maximum and as a result to primary particle type. In this paper, we developed a parametric method of separation between heavy and light groups of nuclei using the 'knee-like' approximation of LDF and taking into account measurement uncertainty. 


\section{Introduction}

Cosmic rays (CR) are charged particles of extraterrestrial origin impinging on the Earth's atmosphere. They are mostly made up of protons, with a small fraction of other fully ionized nuclei and electrons. The CR energy spectrum can be described by a power law with two breaks: the knee (steepening at a few PeV) and the ankle (flattening at a few EeV). The chemical composition of cosmic rays in the energy range below the knee is a keystone in understanding the origin of the knee. However, the volume of direct experimental data in the energy range 300-3000 TeV is very limited. On the other hand, for most EAS experiments the region lower than $1000 \mathrm{TeV}$ is below the energy threshold. The HiSCORE (High Sensitivity Cosmic Ray and gamma-ray Explorer) wide angle Cherenkov array, which is a part of the TAIGA experiment [1-3], allows initiating this study, because at energy above $300 \mathrm{TeV}$ the amount of tens of millions of events can be collected during one year. The lateral distribution of Cherenkov light from EAS initiated by various primary particles can be measured by the highly sensitive HiSCORE stations. They also detect EAS front arrival times (with a ns accuracy) $[4,5]$. In this paper, we developed a parametric method of separation between heavy and light groups of nuclei using the 'knee-like' approximation of LDF [6-8] and taking into account measurement uncertainty.

As it was shown previously, many years ago $[9,10]$, a full steepness of LDF is sensitive to the depth of shower maximum and as a result to primary particle type. In this paper, we developed a parametric method of separation between heavy and light groups of nuclei using the 'knee-like' approximation of LDF [6-8]. Simulation with CORSIKA revealed which approximation parameters are most sensitive to primary particle type. Measurement uncertainties were estimated and introduced in Monte Carlo simulation. For preliminary testing of the approach we used data (one day of exposition) obtained in the TAIGA-HiSCORE experiment during the season 2016-2017.

\section{Parameters of LDF being sensitive to sort of primary particles}

In our previous works [6-8] we developed a fitting routine for lateral distribution of Cherenkov light $Q(R)$ using the so called 'knee-like approximation', the name reflecting the presence of a plateau-like peak (in double logarithmic scale) with a position of the knee determined by the Cherenkov angle and shower maximum depth. We studied the properties of the knee-like fitting and its applications using CORSIKA simulations in [8-10], earlier [11] this function was used for description of the knee in the cosmic 
ray spectrum, $F(E)$. It has five free parameters $C, \gamma_{1}, \gamma_{2}$, Ro and $\alpha$, where $R 0$ is the knee position, $\gamma_{1}$ is the slope of the LDF below the knee, and $\gamma_{2}+\gamma_{1}$ is the slope of the LDF above the knee. Parameter $\alpha$ determines the sharpness of the knee.

$$
F(R)=C R^{\gamma 1}\left(1+(R / R 0)^{\alpha}\right)^{\gamma 2 / \alpha}
$$

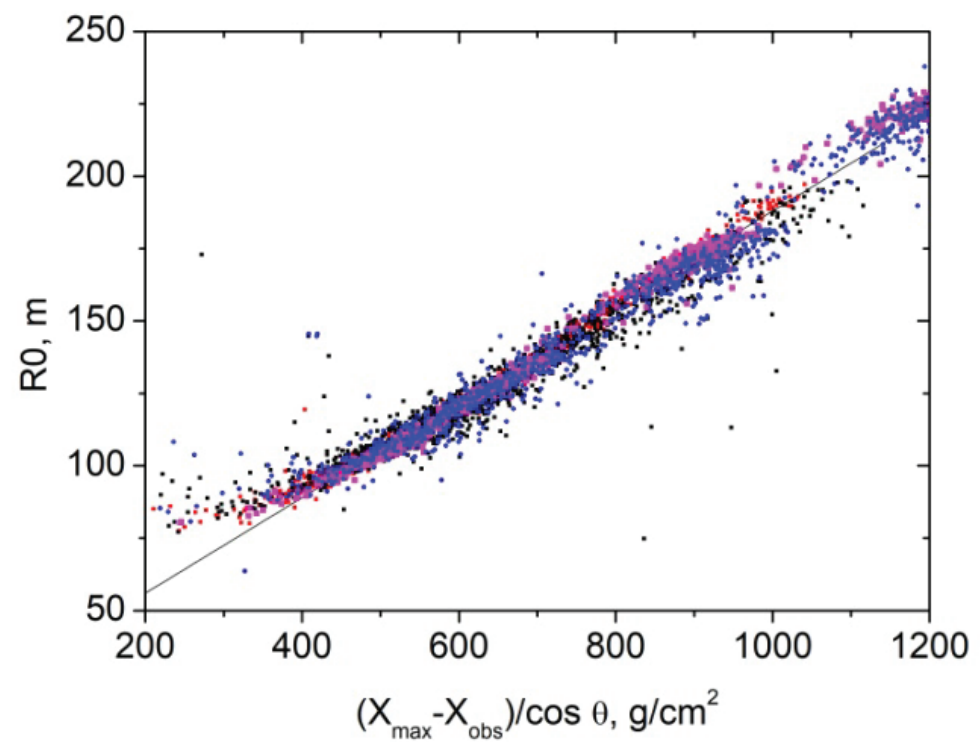

Figure 1: Dependence of $R 0$ on the distance to the shower maximum $\left(X_{\max }-X_{o b s}\right) / \cos \theta$ for different primary particle types, denoted by different colours.

We started this work using Monte Carlo simulated data, and have finally revealed that the most sensitive to primary particle type parameter is $R 0$. At energy $300 \mathrm{TeV}$, $\mathrm{R}_{0}$ shows an almost linear dependence on the distance to the shower maximum $\left(X_{\max }\right.$ $X_{o b s}$ ) $/ \cos \theta$ (Figure 1 ) for 4 primary particle types from gamma to proton to Fe. Parameters $\gamma_{1}$ and $\alpha$ are also sensitive to primary particle type. However, further study showed that the accuracy of free parameters estimation depends on the accuracy of $Q(R)$ reconstruction in experiment. Therefore, we need to take into consideration all details of $Q(R)$ measurement procedure as well as the event reconstruction process.

\section{Experimental procedure and Monte Carlo simulations}

We use the experimental sample of showers accumulated in the TAIGA-HiSCORE experiment during one day 01.02. 2017. The TAIGA-HiSCORE array is under development; during the season 2016-2017 it had 28 stations operational, each with a field of view of $0.6 \mathrm{sr}$ and a light collecting area $0.5 \mathrm{~m}^{2}$, placed $106 \mathrm{~m}$ apart and distributed over $0.25 \mathrm{~km}^{2}$. Only events with the number of triggered stations greater than 15 were selected for the analysis of the experimental LDF. The standard procedure of core 
position and arrival direction reconstruction $[4,5]$ was applied to the selected events, therefore, in each event we calculated the distance of each station to the shower core $R$ (in the shower plane). These points were approximated by the 'knee-like' fitting function (1) to obtain fitting parameters: $R_{0}, \gamma_{1}, \gamma_{2}, \alpha$. The accuracy of LDF parameters estimation depends on the absolute threshold of the Cherenkov photon number detection, and on accuracy of $Q$-value measurement. We carried out an additional study of the distribution of experimental points deviation from fit, dlgQ, for small intervals of $Q$. Our study showed that for small intervals of $Q$ the distribution $F(d \lg Q)$ can be adequately approximated by a normal distribution whose standard deviation, sigma, falls rapidly with an increase in $Q$ value. The appropriate distortion function was developed and added to the MC simulations. To test the method, we selected experimental and Monte Carlo simulated events that satisfy the following conditions: the energy 800-1300 TeV, azimuth angle $\theta<25^{\circ}$, the number of hit detectors $N_{\text {hit }}>13$. Seeing the $R 0$ parameter strong dependence on the distance to the shower maximum, its dependence on primary particle type is worth considering only for events within a small angular interval.

\section{Parametric approach to discrimination between groups of nuclei}

When we add experimental uncertainties in $Q(R)$ reconstruction to the $M C$ simulations, we realize that the difference between $R_{0}$ for the two groups of nuclei, heavy and light ones, become smaller. For that reason we use for selection two parameters simultaneously, $R O$ and $\gamma_{1}$, the latter reflecting the steepness of the LDF and also sensitive to primary particle type. In Figure 2 (left) we present the scatter plot of parameters $R_{0}$ and $\gamma_{1}$ for two groups of primary nuclei: $\mathrm{Pr}+\mathrm{He}$ and $\mathrm{CO}+\mathrm{Fe}$. We calculated the number of events between the lines (in the rotated coordinate system) and draw its histogram for the both groups, Figurez (right).

At the next step we tested different (from 0.1 to 0.9) proportions of light nuclei to all events, calculating the Chi2 value for each case. The best agreement between experiment and simulation was found for the proportion 0.5-0.7. In Figure 3 we show the histogram for experimental and simulated events with the optimal proportion of light nuclei $(50 \%)$.We see that the mixed composition of primary nuclei at energies around $1000 \mathrm{TeV}$ is preferred. An abrupt change of composition in this region has a small probability. 

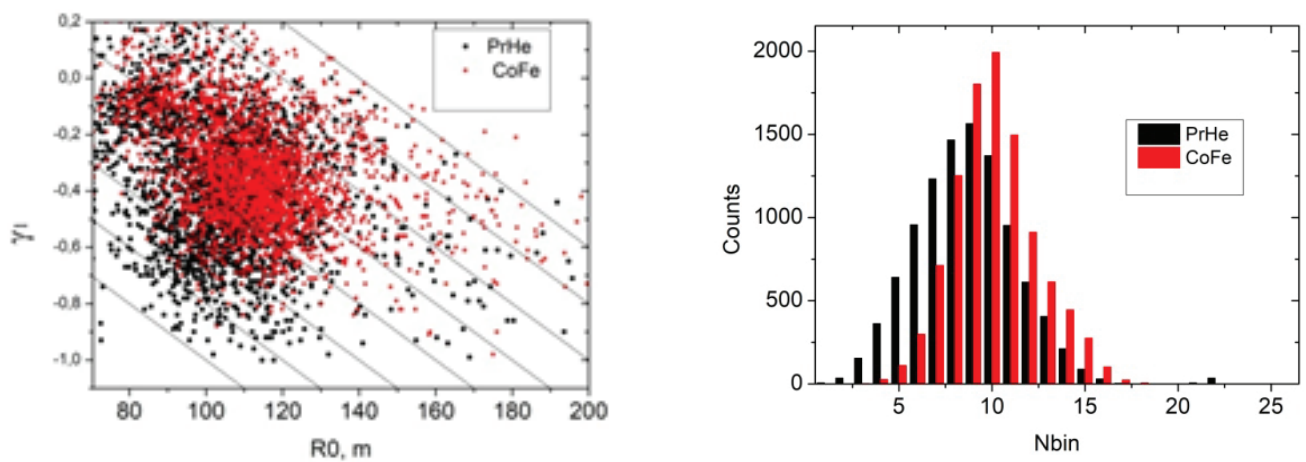

Figure 2: Left: scatter plot of parameters Ro and $\gamma_{1}$ for two groups of primary particles $\mathrm{Pr}+\mathrm{He}$ (black points) and $\mathrm{CO}+\mathrm{Fe}$ (red points). Right: histograms of the number of events located between the lines of the left plot.
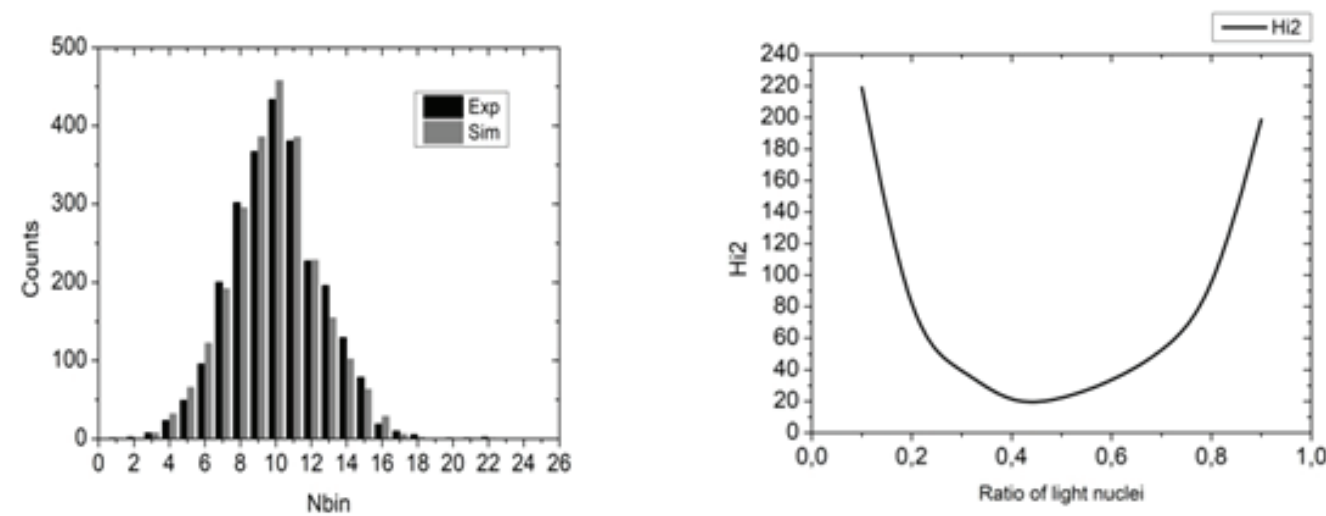

Figure 3: Left: histograms of the number of events located between the lines of the left plot of Figure 2 for experimental (black) and simulated events with 50\% proportion of light nuclei (grey). Right: Chiz dependence (for 24 bins) on the proportion of light nuclei.

\section{Conclusion}

We propose a method of separation between light $(\mathrm{Pr}+\mathrm{He})$ and heavy $(\mathrm{CO}+\mathrm{Fe})$ groups of primary nuclei. It's based on parametric analysis of lateral distribution of Cherenkov light in the atmosphere. LDF are approximated by the 'knee-like' fitting function (1) proposed earlier in [6-8]. We used a tested sample of experimental events collected during one day of observation at the TAIGA-HiSCORE observatory in the energy range around $1000 \mathrm{TeV}$, and obtained a good agreement between experiment and MC simulation. At this stage we could not draw a conclusion about mass composition of cosmic rays in the energy range 300-1000 TeV, but we hope to achieve this aim after refinement of the method. 


\section{Acknowledgments}

The work was supported by the Russian Federation Ministry of Education and Science (14.593.21.0005 (support of the core facilities) and 3.10131.2017/NM, 3.9678.2017/8.9, 3.904.2017/4.6), the Russian Foundation for Basic Research (grant 16-29-13035, 16-0200738) and the grant 15-12-20022 of the Russian Science Foundation (section 3).

\section{References}

[1] Mirzoyan R 2014 40 th COSPAR Scientific Assembly (Moscow) V 40

[2] Kuzmichev L et al (TAIGA collaboration) EPJ Web of Conf. 14501001

[3] Tluczykont M, Hampf D, Horns D, Spitschan D, Kuzmichev L, Prosin V, Spiering C, Wischnewski R 2014 Astroparticle Physics 56421992

[4] Prosin V V, Berezhnev S F et al 2014 Nucl. Instrum. Methods Phys. Res., Sect. A 694

[5] Prosin V V, Berezhnev S F, Budnev N M et al 2015 EPJ Web of Conferences 9904002

[6] Elshoukrofy A, Postnikov E et al 2017 Bull. Russ. Acad. Sci. Phys. 4453

[7] Elshoukrofy A Postnikov E, Sveshnikova L 2017 J. of Phys.: Conf. Ser. 798012184.

[8] Elshoukrofy A, Postnikov E, Korosteleva E, Sveshnikova L 2017 EPJ Web of Conf. 145 19006

[9] J. R. Patterson and A. M. Hillas 1983 J. of Physics G Nuclear Physics 91433

[10] Dawson B R et al 1989, J. of Physics G Nuclear Physics 15893 